\title{
Trial shows caries reductions at one year in school-based sealant programme
}

\author{
Abstracted from \\ Muller-Bolla M, Lupi-Pegurier L, Bardakjian H, Velly AM. \\ Effectiveness of school-based dental sealant programs among children from \\ low-income backgrounds in France: a pragmatic randomized clinical trial. \\ Community Dent Oral Epidemiol 2013; 41: 232-241. \\ Address for correspondence: Michèle Muller-Bolla, Faculte 'de Chirurgie Dentaire, \\ 24, Avenue des Diables Bleus, 06357 Nice Cedex 4, France. E-mail: michele.muller@unice.fr
}

\section{Question: Is a school-based sealant programme effective in reducing caries?}

Design Pragmatic split-mouth randomised controlled trial. Intervention Children from low-income backgrounds were randomised to have one of each first molar tooth pairs sealed. Tooth pairs were excluded when a dental sealant or dentinal caries was present on one of the teeth. Clinical examinations took place at baseline and at one year. Caries risk status was assessed at baseline and included Streptococcus mutans (SM) and Lactobacillus counts. Outcome measure The primary outcome was new carious lesions (ICDAS code 3-6). Secondary outcomes included sealant status. Results 343 children consented, 253 (91.7\%) of the 276 children who had sealants were seen at one-year giving 421 tooth pairs for analysis. The adjusted analysis showed that seared molars were less at risk of developing new caries compared with controls ( $\mathrm{OR}=0.26,95 \% \mathrm{Cl}$ : 0.14 0.49). The effect of the sealants was significant only when the analyses included subjects with active caries (OR $=0.25 ; 95 \% \mathrm{Cl}: 0.12-0.50)$ or with a high SM count ( $>105, \mathrm{OR}=0.20 ; 95 \% \mathrm{Cl}: 0.10-0.41)$ at baseline. Total retention was recorded in $52.7 \%(n=222)$ of the treated teeth, with retention in the maxilla being significantly lower than in the mandible. Conclusions The one-year effectiveness of the school-based program was demonstrated in low socio-economic areas. Selection of schoolchildren according to individual caries risk factors should be considered in school-based programmes.

\section{Commentary}

Systematic reviews provide clear evidence about the usability of dental sealants in the prevention of occlusal caries. This study is an effort to strengthen the evidence for sealants in a school-based program (SBDS). The reported trial has all the vital components of a RCT, is well-structured and well reported.

The children were recruited with fewer eligibility restrictions, which broadens the generalisability of the results. It has a randomised split-mouth design which ensures similarity among the groups at baseline, avoiding the effect of confounders.

The allocation sequence was generated using the sequence of four; such a small block may lead to predictability of the allocation at the end of each block leading to selection bias. The investigator responsible for generation of allocation sequence also assigned the participants to intervention, raising the concern for bias. Probably the assignment schedule was locked away but has not been reported.
One or both of the pairs of permanent first molars were selected; a randomised tooth from the pair was treated with sealant while the other served as control. After one year the effectiveness of sealants was evaluated in preventing new caries. The association between baseline individual caries risk (ICR) factors and caries outcome and sealant retention was also evaluated. Different authors were responsible for evaluating the caries at baseline and after one year. They showed good intra-examiner reliability while inter-examiner reliability is not reported.

Among all the participants ( $\mathrm{n}=276), 23$ were not included in the final analysis. The results were analysed by intention to treat; this approach is an integral part of a pragmatic trial, is less likely to lead to bias and more closely reflects the impact of treatment. The authors undertook an aggressive approach as they performed a secondary analysis by imputing the missing data outcomes with 'presence of caries' $=1$. The secondary analysis thus included all the tooth pairs, $n=457$.

They concluded that the permanent molars had significantly less risk of developing new caries as compared to control $(\mathrm{OR}=0.26, \mathrm{CI}=$ 0.14-0.49). Among all ICR factors only 'active caries at baseline' was significantly related to new caries at one year. Furthermore, a significantly decreased risk was observed when the analyses included subjects with active caries or high SM count at baseline. The authors thus demonstrated that the sealant effectiveness is more pronounced among children with active caries and high SM counts at baseline. Hence authors emphasised that selection of children as per ICR factors should be considered in a SBDS program while they also argued that there is lack of definitive consensus regarding the risk factors that should be used for ICR assessment. Conclusively, in spite of the low sealant retention observed at one year, this randomised split-mouth study demonstrates significant reduced new caries lesions among the treated group and can be used as a reference by different health authorities to implement such a program in areas where access to dental care is limited.

\section{Practice point}

- Use of sealants significantly reduces the risk of developing new caries in sealed teeth

- Sealant effectiveness is more pronounced among 'High Caries Risk Group' children, assessed by Individual caries risk factors assessment.

Neeraj Gugnani

Department of Paedodontics \& PCD, DAV $(C)$ Dental College, Haryana, India

Evidence-Based Dentistry (2013) 14, 71. doi:10.1038/sj.ebd.6400946 